Munster, B.C. van, Bisschop, P.H., Zwinderman, A.H., Korevaar, J.C., Endert, E., Wiersinga, W.J., Oosten, 闭住. van, Goslings, J.C., Rooij, S.E. de. Cortisol, interleukins and S100B in delirium in the elderly. Brain and Cognition: 2010, 74(1), 18-23

\begin{tabular}{|l|l|}
\hline Postprint Version & 1.0 \\
\hline Journal website & http://dx.doi.org/10.1016/j.bandc.2010.05.010 \\
\hline Pubmed link & http://www.ncbi.nlm.nih.gov/pubmed/20580479 \\
\hline DOI & $10.1016 /$ j.bandc.2010.05.010 \\
\hline
\end{tabular}

This is a NIVEL certified Post Print, more info at http://www.nivel.eu

\title{
Cortisol, interleukins and S100B in delirium in the elderly
}

BARBARA C. VAN MUNSTER ${ }^{A, B}$ PETER H. BISSCHOP ${ }^{\mathrm{B}}$, AEILKO H. ZWINDERMAN ${ }^{\mathrm{A}}$, JOHANNA C. KOREVAAR $^{\mathrm{A}}$, ERIK ENDERT ${ }^{\mathrm{C}}$, W. JOOST WIERSINGA ${ }^{\mathrm{B}}$, HANNAH E. VAN OOSTEN ${ }^{\mathrm{B}}$, J. CAREL GOSLINGS ${ }^{\mathrm{D}}$ AND SOPHIA E.J.A. DE ROOIJ ${ }^{\mathrm{B}}$

${ }^{a}$ Department of Clinical Epidemiology, Biostatistics and Bioinformatics, Academic Medical Centre, University of Amsterdam, P.O. Box 22660, 1100 DD Amsterdam, The Netherlands

${ }^{b}$ Department of Medicine, Academic Medical Centre, University of Amsterdam, P.O. Box 22660, 1100 DD Amsterdam, The Netherlands

${ }^{\mathrm{c}}$ Department of Clinical Chemistry, Laboratory of Endocrinology and Radiochemistry, Academic Medical Centre, University of Amsterdam, P.O. Box 22660, 1100 DD Amsterdam, The Netherlands

d Trauma Unit Department Surgery, Academic Medical Centre, University of Amsterdam, P.O. Box 22660, 1100 DD Amsterdam, The Netherlands

\begin{abstract}
In independent studies delirium was associated with higher levels of cortisol, interleukin(IL)s, and S100B. The aim of this study was to simultaneously compare cortisol, IL-6, IL-8, and S100B levels in patients aged 65 years and older admitted for hip fracture surgery with and without delirium. Cortisol, IL-6, IL-8, and S100B were assayed in repeated blood samples. 120 patients (mean age 84 years, 62 patients with delirium) were included. Highest levels of IL-8 (27.1, 95\% Confidence Interval (CI): $13.6-53.1 \mathrm{pg} / \mathrm{ml})$ and cortisol $(666,95 \% \mathrm{CI}: 475-$ $859 \mathrm{nmol} / \mathrm{L})$ were before delirium, but of IL-6 (84.3, 95\% CI: $46.5-151.4 \mathrm{pg} / \mathrm{mL})$ and S100B $(0.18,95 \%$ CI: $0.12-0.24 \mu \mathrm{g} / \mathrm{L})$ during delirium. In multivariable analysis cortisol, LogIL-6, and LogS100B were significantly associated with delirium, but adjusted for pre-existing cognitive impairment, only $\operatorname{LogS100B}$ remained significantly associated. Cortisol, IL-6 and S100B may have a role in the pathogenesis of delirium, but S100B is the strongest independent marker.
\end{abstract}

\section{INTRODUCTION}

Delirium is one of the most frequent psychiatric syndromes in the elderly, especially in patients with preexistent cognitive impairment (Young \& Inouye, 2007). The incidence of post-operative delirium following orthopedic surgery for hip fracture varies between $16 \%$ and $62 \%$ with a mean duration of 3 days (Bitsch, Foss, Kristensen, \& Kehlet, 2004). It is independently associated with an increase in mortality, impaired physical and cognitive recovery, and increased hospital costs. Despite these serious effects on outcome, the pathogenesis of delirium is still incompletely understood (Maclullich, Ferguson, Miller, de Rooij, \& Cunningham, 2008). Stress hormones as well as proinflammatory cytokines can affect aspects of mental function like attention, memory, mood, and perception (Brydon et al., 2009). With ageing and neurodegeneration, dysfunction of the stress response and heightened inflammatory states are common even under normal conditions ([Eikelenboom et al., 2002] and [Luz et al., 2003]). Moreover, an exaggerated inflammatory response and sustained higher cortisol levels due to stimulation of these systems 


\section{Munster, B.C. van, Bisschop, P.H., Zwinderman, A.H., Korevaar, J.C., Endert, E., Wiersinga, W.J., Oosten, 用优.V van, Goslings, J.C., Rooij, S.E. de. Cortisol, interleukins and S100B in delirium in the elderly. Brain and Cognition: 2010, 74(1), 18-23}

by illness or surgery has been found in older people or people with neurodegenerative disease ([Eikelenboom and Hoogendijk, 1999], [Godbout and Johnson, 2006] and [Maclullich et al., 2008]).

Since both stress hormones as cytokines affect mental functioning, it has been hypothesized that the interaction of the enhanced response of the stress hormone and inflammation system in older patients with damaged neurons finally lead to delirium (Maclullich et al., 2008). In this respect could the higher risk of long-term cognitive impairment after a delirious episode reflect the detrimental effects of the above mechanisms on the brain or the inappropriate continuation of sickness behaviour ([Cunningham et al., 2008] and [Viljoen and Panzer, 2005]). A marker of brain damage used in various diseases is S100Bprotein (S100B) (Goncalves, Leite, \& Nardin, 2008). S100B belongs to the family of calcium binding proteins, is expressed mainly by astrocytes and is found both intra- and extracellularly in brain tissue. It is usually elevated due to nervous system damage in CSF as well as in blood, due to functional disturbance of membrane integrity and/or increased permeability of the blood-brain barrier. Elevated levels of S100B, a marker of cerebral damage, were found to be associated with delirium in medical patients (van Munster et al., 2009), in patients after abdominal surgery (Rasmussen, Christiansen, Rasmussen, Kristensen, \& Moller, 2000), after hip fracture surgery (van Munster et al., 2009), after cardiac surgery (Herrmann et al., 2000) and sepsis-associated delirium (Pfister et al., 2008).

In humans, the most investigated proinflammatory cytokines in delirium are Interleukin (IL)-6 and IL-8. Inconsistent associations with delirium have been shown (Marcantonio et al., 2006). Higher levels of Interleukin (IL)-6 in the CSF were found in Systemic Lupus Erythematosus patients with delirium (Katsumata et al., 2007), and in blood in elderly patients with post-operative confusion (Kudoh, Takase, Katagai, \& Takazawa, 2005), post-operative delirium (van Munster et al., 2008) and in medical patients (de Rooij, van Munster, Korevaar, \& Levi, 2007). However, in another study the higher level of IL-6 was not confirmed in medical patients with delirium Adamis et al., 2007) and no relation was found between preoperative measured IL-6 and post-operative delirium in elderly hip fracture patients (Lemstra, Kalisvaart, Vreeswijk, Van Gool, \& Eikelenboom, 2008). A difference in the time-course of IL-8 was demonstrated in hip fracture patients with delirium (Beloosesky et al., 2007), but higher levels of IL-8 were not found in patients with delirium after cardiac surgery (Rudolph et al., 2008). Only small studies investigated the relationship between stress hormones and delirium, but they consistently showed that an activated hypothalamic-pituitary-adrenal (HPA) axis was associated with delirium in patients after surgery ([Kudoh et al., 2005] and [McIntosh et al., 1985]), in medical patients (O’Keeffe \& Devlin, 1994) and in demented patients with delirium (Robertsson et al., 2001).

To elaborate on the above hypothesis, we were interested in the association of the stress and inflammatory system in elderly patients with delirium and the damaging effect of the processes leading to delirium on the brain. Previously, associations of cortisol, interleukins, and S100B with delirium were determined in separate independent studies. We hypothesize that levels of cortisol, IL-6, IL-8 and S100B are simultaneously increased during delirium. Therefore, the aim of this study was to investigate the levels of cortisol in a large sample and compare the levels cortisol, IL-6 and IL-8 and S100B in one study among elderly patients with hip fracture with and without delirium.

\section{MATERIALS AND METHODS}

\subsection{Patient population}

From May 2005 through February 2008, consecutive patients 65 years of age or older who were acutely admitted with hip fracture and scheduled for operation at the Department of Orthopedic Surgery or Trauma Surgery of the Academic Medical Centre, Amsterdam were invited to participate. Informed consent was obtained from all patients or substitute decision-makers in cases of cognitive impairment. Patients were excluded if they were unable to speak or understand Dutch or English. The institutional Medical Ethics Committee approved the study.

\subsection{Procedures}

Two geriatricians, a fellow in geriatric medicine, and four research nurses trained in geriatric medicine collected demographic and clinical data. The presence or absence of delirium was scored during weekdays by a physician and a nurse using the Confusion Assessment Method (CAM) (Inouye et al., 1990). We based our information for the CAM on our psychiatric examination of the patient, medical and nursing records 


\section{Munster, B.C. van, Bisschop, P.H., Zwinderman, A.H., Korevaar, J.C., Endert, E., Wiersinga, W.J., Oosten, 用优Ve van, Goslings, J.C., Rooij, S.E. de. Cortisol, interleukins and S100B in delirium in the elderly. Brain and Cognition: 2010, 74(1), 18-23}

including the Delirium Observation Screening Scale of three shifts (DOS) (Schuurmans, ShortridgeBaggett, \& Duursma, 2003), and information given by the patient's closest relative. When there was disagreement about the diagnosis between nurse and geriatrician, the patient was discussed in the geriatric consultation team to gain consensus. Medical and nursing records were checked for delirious symptoms in between days of blood withdrawal.

Possible confounding factors, including demography, fracture characteristics, type of anesthesia, type of surgery, peri- and post-operative complications (including infectious events), were registered for all patients. We scored the presence of infection retrospectively by positive cultures and the start of antibiotics. Severity and number of comorbidities were scored with the Charlson comorbidity index (Charlson, Pompei, Ales, \& MacKenzie, 1987). Pre-existent global cognitive functioning was based upon anamnesis, medical history, and Informant Questionnaire on Cognitive Decline short form (IQCODE-sf) (Jorm, 1994). The informant was asked to recall the situation 2 weeks prior to the hip fracture and compare it with the situation 10 years earlier. Patients with a mean score of 3.9 or higher were considered to have global cognitive impairment (de Jonghe, 1997). To measure pre-existent physical functionality, we asked patients, or their closest relative in cases of cognitive impairment, to complete the 15-item Katz ADL scale based on the situation 2 weeks prior to the hip fracture (Weinberger et al., 1992). Patients with a score of seven and more were considered as functional impaired.

A maximum of four blood samples was collected during the hospital stay under similar conditions at 11.00 am during weekdays (see Supplementary Table 1). Blood was kept on ice after withdrawal. Serum and ethylenediaminetetraacetic acid plasma was obtained by centrifugation for $15 \mathrm{~min}$ at $1780 \mathrm{~g}$ at $4{ }^{\circ} \mathrm{C}$, and aliquots were stored at $-80^{\circ} \mathrm{C}$. Cortisol was assayed on an Immulite system (Siemens Healthcare Diagnostics B.V., Breda, The Netherlands). Samples below the detection limit were set at $45 \mathrm{nmol} / \mathrm{L}$. IL-6 and IL-8 concentrations were measured in plasma using a cytometric bead array immunoassay (BD Biosciences Pharmingen, San Diego, CA). Levels below $10 \mathrm{pg} / \mathrm{mL}$ for IL-6 and below $20 \mathrm{pg} / \mathrm{mL}$ for IL-8 were considered suboptimal, because of the limits for reliable detection. Nevertheless, these original cytokine values of the CBA assay were analyzed since they give a more accurate estimation of the real sample value than imputation of the detection level or any other imputed value might give. S-100B levels were measured in serum on the Modular Analytics E170 (Elecsys module) analyzer (Roche Diagnostics, Mannheim, Germany) using the electrochemiluminescence immunoassays (ECLIA) technique. Normal values were established before as below $0.10 \mu \mathrm{g} / \mathrm{L}$. Levels below the detection limit of $0.020(\mu \mathrm{g} / \mathrm{L})$ were set at 0.020 .

\subsection{Statistical analysis}

Statistical Package for the Social Sciences (SPSS) version 15.0 was used for data analysis. We tested for differences in characteristics in patients with and without delirium using T-tests and Mann-Whitney tests. Variables that were not normally distributed were expressed as median scores and inter-quartile ranges. A two-tailed criterion of $p<0.05$ was considered statistically significant. Correlations between cortisol, IL-6, IL-8 and S100B were determined using MANOVA, with adjustment for the repeating measuring the same patient and with adjustment for a shared effect of time since surgery.

To fulfil the assumption of normality of the distribution of the IL-6, IL-8 and S100B values, we had to perform a log transformation of the original value. To examine the association of cortisol, Log IL-6, Log IL-8 and Log S100B with delirium state we used the linear mixed models approach with these factors as dependent variables in four separate analyzes. 'Delirious state' consisted of four categories based on the CAM scores of the patient at the moment the sample was taken: samples from patients in the non-delirious group were all categorized (1) 'non-delirious'. Samples from patients in the delirious group were categorized (2) 'before delirium', (3) 'during delirium' or (4) 'after delirium', based on the individual CAM scores of the patients the samples were taken. Patient number was taken as a random effect, and day of sampling (counted from the day of surgery) and delirious state were taken as fixed effects. In addition, preor post-operative status was taken as covariate. We selected the samples between the day before surgery and 8 days post surgery. The goodness of fit of the mixed models was inspected by assessing the distribution of the residuals. The analysis was repeated adding age, infection, pre-existent cognitive and functional impairment as covariates.

Subsequently, the four factors were univariable and multivariable analyzed through logistic regression using generalized estimating equations with delirium as dependent variable, and cortisol and the e-based 


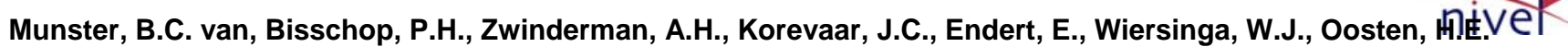 van, Goslings, J.C., Rooij, S.E. de. Cortisol, interleukins and S100B in delirium in the elderly. Brain and Cognition: 2010, 74(1), 18-23}

$\log$ transformed IL-6, IL-8 and S100B value as independent variables. In a GEE analyses all measurements of all individual patients are used, taking into account which measurements originate from one patient. For this analysis we included the samples taken during delirium of the patients with delirium and excluded the samples taken before surgery. In a backward selection, all variables with p-value above 0.05 were discarded from the logistic model.

\section{RESULTS}

During the inclusion period, 241 patients aged 65 years and older were admitted and fulfilled the inclusion criteria. A total of 54 patients were excluded due to absence of informed consent and 67 patients did not consent to blood withdrawal. Selected and non-selected patients were not significantly different with respect to age but there were more men in the included patients (33\% vs. $21 \%$ ). In total 120 patients were included, 62 (52\%) with delirium and 58 without. This resulted in 377 samples in total, 192 samples of the delirious patients ( 21 samples before the delirious episode, 117 samples during the delirious episode, 54 after the delirious episode) and 185 samples of the patients without delirium. Mean day of blood withdrawal of all samples was 3 (IQR 1-5), and 58 samples were taken pre-operatively.

Characteristics of the 120 included patients are presented in Table 1. Patients with delirium experienced pre-existing cognitive and functional impairment significantly more often and were significantly more likely to live in either old-people's homes or nursing homes than patients without delirium. There was no significant difference in the total number of complications $(p=0.07)$ or concurrent infections $(p=0.37)$ between patients with and without delirium. The median length of stay was 14 days for patients with delirium and 11 days for patients without delirium $(p=0.06)$.

\section{[TABLE 1]}

An overview of the median levels of the day of surgery before the surgical procedure and the first 5 days post surgery is shown in Fig. 1 of cortisol (a), IL-6 (b), IL-8 (c), and S100B (d). Significant correlations existed between Log IL-6 and Log IL-8 $(r=0.59, p<0.001)$, Log IL-6 and Log S100B $(r=0.30$, $\mathrm{p}<0.001)$ and Log IL-8 and Log S100B $(r=0.30, \mathrm{p}<0.001)$.

\section{[FIGURE 1]}

The linear mixed model showed that cortisol, IL-6, IL-8 and S100B were all significantly associated with overall 'delirious state' $(\mathrm{p}<0.01)$ (Table 2). Samples drawn of patients without delirium had the lowest value compared to all samples drawn of the patients with delirium for all factors. Although not significant, a trend was seen for higher levels during the delirious episode of IL-6 (84.3, 95\% C.I. 46.5-151.4 pg/mL) and S100B (0.18, 95\% C.I. 0.12-0.24 $\mu \mathrm{g} / \mathrm{L})$, but of IL-8 (27.1, 95\% C.I. 13.6-53.1 pg/mL) and cortisol (666 95\% C.I. 475-859 nmol/L) levels were highest before delirium. IL-6, IL-8 and S100B levels were influenced by surgery, but levels of cortisol were not $(p=0.58)$. Repeating the mixed model analysis of cortisol in the women only did not influence the results. Mixed model analysis adjusted for age, infection, cognitive and functional impairment showed that IL-8 and cortisol were significantly associated with age. The other variables showed no association with cortisol, IL-6, IL-8 and S100B.

\section{[TABLE 2]}

Focusing on the comparison of samples taken during delirium to the samples of patients without delirium, higher levels of cortisol (Odds Ratio (OR): 1.003, 95\% Confidence Interval (CI): 1.001-1.005), $\mathrm{p}=0.01$ ), Log IL-6 (OR: 1.78, 95\% CI: 1.29-2.48, p = 0.001), Log IL-8 (OR: 1.61, 95\% CI: 1.08-2.42, p = 0.02) and Log S100B (OR: 3.43, 95\% CI: 1.78-6.70, $\mathrm{p}<0.001$ ) were measured in samples from patients with delirium (Table 3 ). As some of these factors were significantly correlated, we also looked at the factors combined and found that, except for Log IL-8, all factors were independently associated with delirium. However, if we added pre-existing cognitive impairment to the model, only Log S100B (OR: 4.0 95\% CI: $1.9-8.6, \mathrm{p}<0.001$ ) was still significantly associated with delirium. 
Munster, B.C. van, Bisschop, P.H., Zwinderman, A.H., Korevaar, J.C., Endert, E., Wiersinga, W.J., Oosten, 用他V van, Goslings, J.C., Rooij, S.E. de. Cortisol, interleukins and S100B in delirium in the elderly. Brain and Cognition: 2010, 74(1), 18-23

\section{[TABLE 3]}

\section{DISCUSSION}

This study among elderly patients admitted for surgical repair of a hip fracture showed that delirium was associated with elevated blood levels of cortisol, IL-6, IL-8 and S100B. Highest levels of IL-6 and S100B were found during delirium, whereas for IL-8 and cortisol the levels were highest before delirium. In combined analysis cortisol, Log IL-6, and Log S100B were independently associated with delirium, but after adjustment for pre-existent cognitive impairment only Log S100B remained significantly associated with delirium. The study population was representative for a normal population of elderly patients admitted with hip fracture. A similar frequency of delirium (52\%) has been found in other large studies of hip fracture patients (Bruce et al., 2007 A.J. Bruce, C.W. Ritchie, R. Blizard, R. Lai and P. Raven, The incidence of delirium associated with orthopedic surgery: A meta-analytic review, International Psychogeriatrics 19 (2007), pp. 197-214. Full Text via CrossRef | View Record in Scopus | Cited By in Scopus (25)Bruce, Ritchie, Blizard, Lai, \& Raven, 2007). The expected risk factors for delirium in surgical patients, such as pre-existing functional and cognitive impairment, were confirmed in our study.

Cortisol, IL-6, and S100B levels are all associated with the development of delirium but not with preexistent cognitive impairment in mixed model analysis. Casual relationship in those four separate models of cross-sectional data remain difficult to interpret. In general, pathophysiological mechanisms cannot be derived from observational studies, but we can speculate about the pathway. Inflammatory mediators such as IL-6 can induce activation of the HPA axis (Maclullich et al., 2008). On the other hand, recent studies showed that chronic stress can also exacerbate inflammatory actions in the brain (Gouin, Hantsoo, \& Kiecolt-Glaser, 2008). Since the level of cortisol seems somewhat higher in patients before delirium, whereas the level of IL- 6 seems highest during delirium this second pathway seems more likely. Importantly, the hypothesis that sustained high levels of cortisol occurring with acute stress are associated with delirium was confirmed in our large study sample.

The correlation between the levels of the proinflammatory cytokines IL- 6 and IL-8 and the level of S100B in the patients in this study is subject to multiple explanations. The raised level of S100B can be either due to cerebral damage caused by multiple different mechanisms (such as hypoxia) leading to neuroinflammation or due to astrocytic overexpression of S100B promoted by high levels of proinflammatory cytokines (Mrak \& Griffin, 2001). On the other hand the raised cytokine levels explained by the heightened inflammatory states in elderly patients with pre-existent cognitive impairment could coexist by coincidence with higher levels of S100B found in dementia ([Dziedzic, 2006] and [Mrak and Griffin, 2001]). After adjusting for pre-existing cognitive impairment in the logistic regression model with delirium, only S100B was still independently associated with delirium. In other words, the independent association with delirium of cortisol and IL-6 could be related to the association of these factors with preexisting cognitive impairment. These data are consistent with literature suggesting an association between peripheral interleukins (Dziedzic, 2006) or cortisol (Magri et al., 2006) and dementia in the peripheral blood. An exaggerated and sustained cortisol response can be important too, since we found higher postoperative cortisol levels. HPA axis dysregulation results in higher basal levels associated with chronic cognitive impairment (Lee et al., 2008) and higher levels of cortisol under conditions of acute stress (Issa, Rowe, Gauthier, \& Meaney, 1990) and these acutely higher levels of cortisol could also be a cause of delirium ([Kudoh et al., 2005] and [Pearson et al., 2010]).

A limitation of our study is the simplified model of markers of inflammation, the stress system and neuronal damage. Firstly, all levels were derived from peripheral blood and may not necessarily reflect the values in the brain. Although, cortisol is sufficient lipophilic to pass the blood brain barrier easily, the hormone is also subject to active transport processes that try to remove the hormone from the brain compartment (Karssen et al., 2001). S100B in blood is lower than that in CSF under normal conditions, but we are not aware of studies that determined S100B in both CSF and blood among patients with cognitive deficits such as delirium (Goncalves et al., 2008). In general, the actual level in the brain is difficult to predict for any of the substances but a correlation with an effect of the relatively high levels in blood on the brain functions seems likely. Secondly, S100B is not a pure marker for cerebral cell damage (Goncalves et al., 2008). The passage of cerebral S100B to serum is modulated by the blood brain barrier (BBB) and therefore serum S100B levels do also reflect the permeability of the BBB. Moreover, except for astrocytes, known S100B-expressing cells are adipocytes, chondrocytes, lymphocytes, bone marrow cells and melanoma cells. Additionally, cortisol at 11.00 am may not be the ideal test for the assessment of HPA axis 
Munster, B.C. van, Bisschop, P.H., Zwinderman, A.H., Korevaar, J.C., Endert, E., Wiersinga, W.J., Oosten, 闸过Ve van, Goslings, J.C., Rooij, S.E. de. Cortisol, interleukins and S100B in delirium in the elderly. Brain and Cognition: 2010, 74(1), 18-23

dysfunction. Diurnal samples and also the dexamethasone suppression test would give additional information regarding acute dysregulation as well as absolute magnitude. Patients with delirium were also shown to have a disturbed dexamethasone suppression test in diverse studies ([O'Keeffe and Devlin, 1994] and [Robertsson et al., 2001]).

A second limitation of our study is that only 120 of the $241(50 \%)$ patients consented to multiple blood withdrawals. Selected and non-selected patients differed with respect to sex; more male patients were included (33\% vs. $21 \%$ ). Selection bias seems not to play a role in the results of S100B and the interleukins, because these factors are not related to gender. Cortisol is higher in woman than in men (Swaab, Bao, \& Lucassen, 2005), but the higher level of cortisol in this study was not attributable to the higher percentage of women in the patients with delirium.

\section{CONCLUSION}

In conclusion, this study in elderly patients with delirium showed that Cortisol, IL-6 and S100B may all have a role in the pathogenesis of delirium, but S100B is the strongest independent marker. Future studies are needed to disentangle the role of each of these factors in the pathway leading to delirium and to investigate the relationship with long-term cognitive impairment. Correlating pre-operative measures of interleukins and cortisol in elective surgery and diurnal measures of these factors to the symptoms over $24 \mathrm{~h}$ period could give some additional information. Understanding the pathophysiology will in the end help to prevent delirium and develop new therapies.

\section{Conflicts of interest}

None

\section{Acknowledgments}

The authors wish to thank the other members of the research team, J.L. Parlevliet, A. Giesbers, J.L. Popma, C.M.M van Rijn, A.C.L. Scheffer, and M.J.A. van der Zwaan. We kindly thank the departments of Orthopaedics and Traumatology for their help in data collection. We are grateful to C.M. Korse, J.M. Bonfrer and E. Delic for determination of S100B.

\section{REFERENCES}

Adamis et al., 2007 D. Adamis, A. Treloar, F.C. Martin, N. Gregson, G. Hamilton and A.J. Macdonald, APOE and cytokines as biological markers for recovery of prevalent delirium in elderly medical inpatients, International Journal of Geriatr Psychiatry 22 (2007), pp. 688-694.

Beloosesky et al., 2007 Y. Beloosesky, D. Hendel, A. Weiss, A. Hershkovitz, J. Grinblat and A. Pirotsky et al., Cytokines and C-reactive protein production in hip-fracture-operated elderly patients, Journals of Gerontology Series A - Biological Sciences and Medical Sciences 62 (2007), pp. 420-426.

Bitsch et al., 2004 M. Bitsch, N. Foss, B. Kristensen and H. Kehlet, Pathogenesis of and management strategies for postoperative delirium after hip fracture: A review, Acta Orthopaedica Scandinavica 75 (2004), pp. 378-389.

Bruce et al., 2007 A.J. Bruce, C.W. Ritchie, R. Blizard, R. Lai and P. Raven, The incidence of delirium associated with orthopedic surgery: A meta-analytic review, International Psychogeriatrics 19 (2007), pp. 197-214.

Brydon et al., 2009 L. Brydon, C. Walker, A. Wawrzyniak, D. Whitehead, H. Okamura and J. Yajima et al., Synergistic effects of psychological and immune stressors on inflammatory cytokine and sickness responses in humans, Brain Behavior and Immunity 23 (2009), pp. 217-224.

Charlson et al., 1987 M.E. Charlson, P. Pompei, K.L. Ales and C.R. MacKenzie, A new method of classifying prognostic comorbidity in longitudinal studies: Development and validation, Journal of Chronic Diseases 40 (1987), pp. 373-383.

Cunningham et al., 2008 Cunningham, C., Campion, S., Lunnon, K., Murray, C. L., Woods, J. F., Deacon, R. M. et al. (2008). Systemic inflammation induces acute behavioral and cognitive changes and accelerates neurodegenerative disease. Biological Psychiatry. 
Munster, B.C. van, Bisschop, P.H., Zwinderman, A.H., Korevaar, J.C., Endert, E., Wiersinga, W.J., Oosten, 闸过Ve van, Goslings, J.C., Rooij, S.E. de. Cortisol, interleukins and S100B in delirium in the elderly. Brain and Cognition: 2010, 74(1), 18-23

de Jonghe, 1997 J.F. de Jonghe, Differentiating between demented and psychiatric patients with the Dutch version of the IQCODE, International Journal of Geriatr Psychiatry 12 (1997), pp. 462-465

de Rooij et al., 2007 S.E. de Rooij, B.C. van Munster, J.C. Korevaar and M. Levi, Cytokines and acute phase response in delirium, Journal of Psychosomatic Research 62 (2007), pp. 521-525.

Dziedzic, 2006 T. Dziedzic, Systemic inflammatory markers and risk of dementia, American Journal of Alzheimers Disease Other Dementia 21 (2006), pp. 258-262.

Eikelenboom and Hoogendijk, 1999 P. Eikelenboom and W.J. Hoogendijk, Do delirium and Alzheimer's dementia share specific pathogenetic mechanisms?, Dementia and Geriatric Cognitive Disorders 10 (1999), pp. 319-324.

Eikelenboom et al., 2002 P. Eikelenboom, W.J. Hoogendijk, C. Jonker and W. van Tilburg, Immunological mechanisms and the spectrum of psychiatric syndromes in Alzheimer's disease, Journal of Psychiatry Research 36 (2002), pp. 269-280.

Godbout and Johnson, 2006 J.P. Godbout and R.W. Johnson, Age and neuroinflammation: A lifetime of psychoneuroimmune consequences, Neurologic Clinics 24 (2006), pp. 521-538.

Goncalves et al., 2008 C.A. Goncalves, M.C. Leite and P. Nardin, Biological and methodological features of the measurement of S100B, a putative marker of brain injury, Clinical Biochemistry 41 (2008), pp. 755763.

Gouin et al., 2008 J.P. Gouin, L. Hantsoo and J.K. Kiecolt-Glaser, Immune dysregulation and chronic stress among older adults: A review, Neuroimmunomodulation 15 (2008), pp. 251-259.

Herrmann et al., 2000 M. Herrmann, A.D. Ebert, I. Galazky, M.T. Wunderlich, W.S. Kunz and C. Huth, Neurobehavioral outcome prediction after cardiac surgery: Role of neurobiochemical markers of damage to neuronal and glial brain tissue, Stroke 31 (2000), pp. 645-650.

Inouye et al., 1990 S.K. Inouye, C.H. van Dyck, C.A. Alessi, S. Balkin, A.P. Siegal and R.I. Horwitz, Clarifying confusion: The confusion assessment method. A new method for detection of delirium, Annuals of Internal Medicine 113 (1990), pp. 941-948.

Issa et al., 1990 A.M. Issa, W. Rowe, S. Gauthier and M.J. Meaney, Hypothalamic-pituitary-adrenal activity in aged, cognitively impaired and cognitively unimpaired rats, Journal of Neuroscience 10 (1990), pp. 3247-3254.

Jorm, 1994 A.F. Jorm, A short form of the Informant Questionnaire on Cognitive Decline in the Elderly (IQCODE): Development and cross-validation, Psychological Medicine 24 (1994), pp. 145-153.

Karssen et al., 2001 A.M. Karssen, O.C. Meijer, D.S.I. Van, P.J. Lucassen, E.C. De Lange and A.G. De Boer et al., Multidrug resistance P-glycoprotein hampers the access of cortisol but not of corticosterone to mouse and human brain, Endocrinology 142 (2001), pp. 2686-2694.

Katsumata et al., 2007 Y. Katsumata, M. Harigai, Y. Kawaguchi, C. Fukasawa, M. Soejima and K. Takagi et al., Diagnostic reliability of cerebral spinal fluid tests for acute confusional state (delirium) in patients with systemic lupus erythematosus: Interleukin 6 (IL-6), IL-8, interferon-alpha, IgG index, and Q-albumin, Journal of Rheumatology 34 (2007), pp. 2010-2017.

Kudoh et al., 2005 A. Kudoh, H. Takase, H. Katagai and T. Takazawa, Postoperative interleukin-6 and cortisol concentrations in elderly patients with postoperative confusion, Neuroimmunomodulation 12 (2005), pp. 60-66.

Lee et al., 2008 B.K. Lee, T.A. Glass, G.S. Wand, M.J. McAtee, K. Bandeen-Roche and K.I. Bolla et al., Apolipoprotein e genotype, cortisol, and cognitive function in community-dwelling older adults, American Journal of Psychiatry 165 (2008), pp. 1456-1464.

Lemstra et al., 2008 A.W. Lemstra, K.J. Kalisvaart, R. Vreeswijk, W.A. Van Gool and P. Eikelenboom, Preoperative inflammatory markers and the risk of postoperative delirium in elderly patients, International Journal of Geriatric Psychiatry 23 (2008), pp. 943-948.

Luz et al., 2003 C. Luz, F. Dornelles, T. Preissler, D. Collaziol, C.I. Da and M.E. Bauer, Impact of psychological and endocrine factors on cytokine production of healthy elderly people, Mechanisms of Ageing and Development 124 (2003), pp. 887-895.

Maclullich et al., 2008 A.M. Maclullich, K.J. Ferguson, T. Miller, S.E. de Rooij and C. Cunningham, Unravelling the pathophysiology of delirium: A focus on the role of aberrant stress responses, Journal of Psychosomatic Research 65 (2008), pp. 229-238.

Magri et al., 2006 F. Magri, L. Cravello, L. Barili, S. Sarra, W. Cinchetti and F. Salmoiraghi et al., Stress and dementia: The role of the hypothalamicpituitary-adrenal axis, Aging Clinical and Experimental Research 18 (2006), pp. 167-170.

Marcantonio et al., 2006 E.R. Marcantonio, J.L. Rudolph, D. Culley, G. Crosby, D. Alsop and S.K. Inouye, Serum biomarkers for delirium, Journal of Gerontology Series A - Biological Sciences and Medical Sciences 61 (2006), pp. 1281-1286. 
Munster, B.C. van, Bisschop, P.H., Zwinderman, A.H., Korevaar, J.C., Endert, E., Wiersinga, W.J., Oosten, 用过Ve van, Goslings, J.C., Rooij, S.E. de. Cortisol, interleukins and S100B in delirium in the elderly. Brain and Cognition: 2010, 74(1), 18-23

McIntosh et al., 1985 T.K. McIntosh, H.L. Bush, N.S. Yeston, R. Grasberger, M. Palter and F. Aun et al., Beta-endorphin, cortisol and postoperative delirium: A preliminary report, Psychoneuroendocrinology 10 (1985), pp. 303-313.

Mrak and Griffin, 2001 R.E. Mrak and W.S. Griffin, The role of activated astrocytes and of the neurotrophic cytokine S100B in the pathogenesis of Alzheimer's disease, Neurobiology of Aging 22 (2001), pp. 915922.

O'Keeffe and Devlin, 1994 S.T. O'Keeffe and J.G. Devlin, Delirium and the dexamethasone suppression test in the elderly, Neuropsychobiology 30 (1994), pp. 153-156.

Pearson et al., 2010 A. Pearson, v.A. de, S.D. Middleton, F. Gillies, T.O. White and I.R. Armstrong et al., Cerebrospinal fluid cortisol levels are higher in patients with delirium versus controls, BMC Research Notes 3 (2010), p. 33.

Pfister et al., 2008 D. Pfister, M. Siegemund, S. I-Kuster, P. Smielewski, S. Ruegg and S.P. Strebel et al., Cerebral perfusion in sepsis-associated delirium, Critical Care 12 (2008), p. R63. Rasmussen et al., 2000 L.S. Rasmussen, M. Christiansen, H. Rasmussen, P.A. Kristensen and J.T. Moller, Do blood concentrations of neurone specific enolase and S-100 beta protein reflect cognitive dysfunction after abdominal surgery? ISPOCD Group, British Journal of Anaesthesia 84 (2000), pp. 242-244.

Robertsson et al., 2001 B. Robertsson, K. Blennow, G. Brane, A. Edman, I. Karlsson and A. Wallin et al., Hyperactivity in the hypothalamic-pituitary-adrenal axis in demented patients with delirium, International Clinical Psychopharmacology 16 (2001), pp. 39-47. Rudolph et al., 2008 J.L. Rudolph, B. Ramlawi, G.A. Kuchel, J.E. McElhaney, D. Xie and F.W. Sellke et al., Chemokines are associated with delirium after cardiac surgery, Journals of Gerontology Series A - Biological Sciences and Medical Sciences 63 (2008), pp. 184-189. Schuurmans et al., 2003 M.J. Schuurmans, L.M. Shortridge-Baggett and S.A. Duursma, The delirium observation screening scale: A screening instrument for delirium, Research Theory Nursing Practice 17 (2003), pp. 31-50. Swaab et al., 2005 D.F. Swaab, A.M. Bao and P.J. Lucassen, The stress system in the human brain in depression and neurodegeneration, Ageing Research Review 4 (2005), pp. 141-194.

van Munster et al., 2008 B.C. van Munster, J.C. Korevaar, A.H. Zwinderman, M. Levi, W.J. Wiersinga and S.E. de Rooij, Time-course of cytokines during delirium in elderly patients with hip fractures, Journal of American Geriatrics Society 56 (2008), pp. 1704-1709.

van Munster et al., 2009 van Munster, B. C., Korevaar, J. C., Korse, C. M., Bonfrer, J. M., Zwinderman, A. H., \& de Rooij, S. E. (2009). Serum S100B in elderly patients with and without delirium. International Journal of Geriatric Psychiatry.

van Munster et al., 2009 B.C. van Munster, C.M. Korse, S.E. de Rooij, J.M. Bonfrer, A.H. Zwinderman and J.C. Korevaar, Markers of cerebral damage during delirium in elderly patients with hip fracture, BMC Neurology 9 (2009), p. 21.

Viljoen and Panzer, 2005 M. Viljoen and A. Panzer, Non-termination of sickness behavior as precipitating factor for mental disorders, Medical Hypotheses 65 (2005), pp. 316-329.

Weinberger et al., 1992 M. Weinberger, G.P. Samsa, K. Schmader, S.M. Greenberg, D.B. Carr and D.S. Wildman, Comparing proxy and patients' perceptions of patients' functional status: Results from an outpatient geriatric clinic, Journal of American Geriatrics Society 40 (1992), pp. 585-588. View Record in Scopus | Cited By in Scopus (92)

Young and Inouye, 2007 J. Young and S.K. Inouye, Delirium in older people, BMJ 334 (2007), pp. 842846.

\section{[TABLES AND FIGURES]}


Munster, B.C. van, Bisschop, P.H., Zwinderman, A.H., Korevaar, J.C., Endert, E., Wiersinga, W.J., Oosten, 闸过Ve van, Goslings, J.C., Rooij, S.E. de. Cortisol, interleukins and S100B in delirium in the elderly. Brain and Cognition: 2010, 74(1), 18-23

\section{Table 1}

Characteristics of patients with and without delirium.

\begin{tabular}{|c|c|c|c|}
\hline Variables & $\begin{array}{l}\text { Delirium } \\
(N=62)\end{array}$ & $\begin{array}{l}\text { No delirium } \\
(N=58)\end{array}$ & $p$-value \\
\hline Age (yrs) & $84.8(6.9)$ & $82.9(7.9)$ & 0.13 \\
\hline Male gender (\%) & $16(26)$ & $23(40)$ & 0.11 \\
\hline Living at home (\%) & $30(48)$ & $51(88)$ & $<0.001$ \\
\hline $\begin{array}{l}\text { Pre-admission functional } \\
\text { impairment (\%) }\end{array}$ & $42(81)$ & $10(19)$ & $<0.001$ \\
\hline $\begin{array}{l}\text { Pre-admission cognitive } \\
\text { impairment (\%) }\end{array}$ & $40(67)$ & $10(18)$ & $<0.001$ \\
\hline Charlson comorbidity index & $6.2(1.8)$ & $5.2(2.3)$ & 0.01 \\
\hline Fracture characteristics (\%) & & & 0.58 \\
\hline Femoral neck fracture & $25(47)$ & $27(48)$ & \\
\hline Intertrochanteric fracture & $33(53)$ & $25(44)$ & \\
\hline Spinal anesthesia (\%) & $22(36)$ & $19(33)$ & 0.35 \\
\hline Type of surgery (\%) & & & 0.64 \\
\hline Internal fixation & $40(65)$ & $35(60)$ & \\
\hline Hip replacement & $22(36)$ & $23(40)$ & \\
\hline Complication (\%) - total & $33(53)$ & $21(37)$ & 0.07 \\
\hline Peri-operative infection & $21(34)$ & $15(26)$ & 0.37 \\
\hline Length of stay - days & $14(8-23)$ & $11(8-14)$ & 0.06 \\
\hline
\end{tabular}

Mean values (SD) are given for continuous variables with a normal distribution, median values (inter-quartile ranges) are given for variables that are not normally distributed. 
Munster, B.C. van, Bisschop, P.H., Zwinderman, A.H., Korevaar, J.C., Endert, E., Wiersinga, W.J., Oosten, 用俌.V| van, Goslings, J.C., Rooij, S.E. de. Cortisol, interleukins and S100B in delirium in the elderly. Brain and Cognition: 2010, 74(1), 18-23
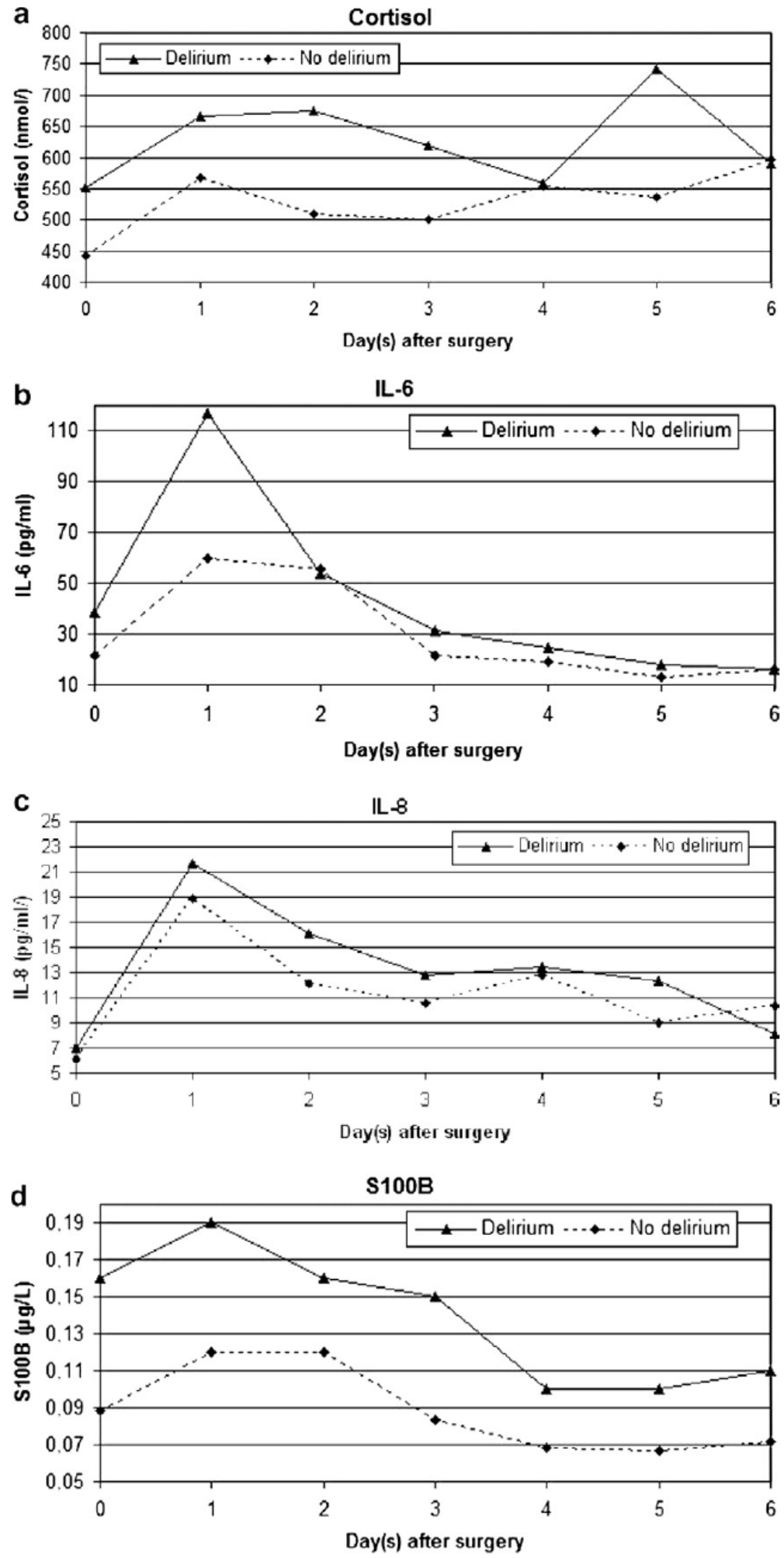

Fig. 1. Measured median levels of cortisol (a), Interleukin (IL)-6 (b), IL-8 (c) and S100B (d) of patients within the first 7 days. Levels of patients with delirium are based on 10 patients day zero (pre-operative), 25 patients day 1,30 patients day 2, 16 patients day 3,12 patients day 4,11 patients day 5 and 5 patients day 6 . Levels of patients without delirium are based on 22 patients day zero (pre-operative), 23 patients day 1,25 patients day 2,32 patients day 3,31 patients day 4,28 patients day 5 and 10 patients day 6 . 
Munster, B.C. van, Bisschop, P.H., Zwinderman, A.H., Korevaar, J.C., Endert, E., Wiersinga, W.J., Oosten, 闸过Vel van, Goslings, J.C., Rooij, S.E. de. Cortisol, interleukins and S100B in delirium in the elderly. Brain and Cognition: 2010, 74(1), 18-23

Table 2

Calculated mean levels (95\% confidence intervals) on the first day after surgery adjusted for post-operative status of cortisol, Interleukin (IL)-6, IL-8 and S100B are shown. Calculations by linear mixed model analysis based on 365 samples drawn of 119 patients (one patients no sample available of this period). p-values are overall values of 'delirious state'

\begin{tabular}{lllll}
\hline & No delirium & Before delirium & During delirium & After delirium \\
\hline Cortisol $(\mathrm{nmol} / \mathrm{L})$ & $517(439-597)$ & $666(475-859)$ & $646(498-795)$ & $584(416-751)$ \\
$\mathrm{IL}-6(\mathrm{pg} / \mathrm{ml})$ & $36.2(27.4-47.9)$ & $63.7(31.8-126.5)$ & $84.3(46.5-151.4)$ & $52.7(39.9-70.1)$ \\
$\mathrm{IL}-8(\mathrm{pg} / \mathrm{ml})$ & $14.7(11.0-19.6)$ & $27.1(13.6-53.1)$ & $21.5(11.7-39.0)$ & 0.002 \\
S100B $(\mu \mathrm{g} / \mathrm{L})$ & $0.12(0.09-0.12)$ & $0.16(0.09-0.22)$ & $0.18(0.12-0.24)$ & 0.010 \\
& & & 0.002 & $0.16(0.4-30.1)$ \\
\hline
\end{tabular}

Table 3

Univariable and multivariable analysis of factors associated with delirium on 295 samples drawn of 111 patients.

\begin{tabular}{|c|c|c|c|c|c|c|}
\hline Factor & Univariable analysis $^{\mathrm{a}}$ & $p$-value & $\begin{array}{l}\text { Multivariable analysis, without } \\
\text { cognitive impairment }^{\mathrm{a}}\end{array}$ & $p$-value & $\begin{array}{l}\text { Multivariable analysis, } \\
\text { with cognitive impairment }^{\mathrm{a}}\end{array}$ & $p$-value \\
\hline Cortisol $(\mathrm{nmol} / \mathrm{L})$ & $1.003(1.001-1.005)$ & 0.01 & $1.002(1.000-1.004)$ & 0.04 & & \\
\hline Log IL-6 (pg/ml) & $1.78(1.29-2.48)$ & 0.001 & $1.42(0.99-2.02)$ & 0.055 & & \\
\hline Log IL-8 (pg/ml) & $1.61(1.08-2.42)$ & 0.02 & & & & \\
\hline $\log \mathrm{S} 100 \mathrm{~B}(\mu \mathrm{g} / \mathrm{L})$ & $3.43(1.78-6.70)$ & $<0.001$ & $2.79(1.39-5.61)$ & 0.004 & $4.01(1.87-8.58)$ & $<0.001$ \\
\hline Pre-existent cognitive impairment & $8.60(3.24-22.8)$ & $<0.001$ & Not included & & $10.5(3.69-29.7)$ & $<0.001$ \\
\hline
\end{tabular}

a Odds ratio ( $95 \%$ confidence interval). 